ANALYSIS OF FIBER SPINNING FOR

THE UPPER-CONVECTED MAXWELL FLUID

By

Thomas Hagen

and

Dias Kurmashev

IMA Preprint Series \# 2291

( December 2009)

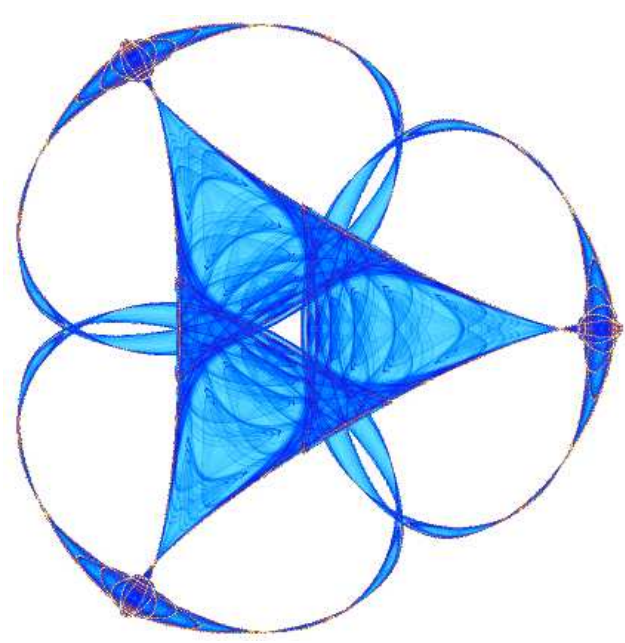

INSTITUTE FOR MATHEMATICS AND ITS APPLICATIONS

UNIVERSITY OF MINNESOTA

400 Lind Hall

207 Church Street S.E.

Minneapolis, Minnesota 55455-0436

Phone: 612-624-6066 Fax: 612-626-7370

URL: http://www.ima.umn.edu 


\title{
Analysis of fiber spinning for the upper-convected Maxwell fluid
}

\author{
Thomas Hagen* and Dias Kurmashev ${ }^{\dagger}$ \\ Department of Mathematical Sciences, The University of Memphis, \\ Memphis, TN 38152, USA
}

December 2009

\begin{abstract}
The fiber spinning process of a viscoelastic liquid modeled by the constitutive theory of the Maxwell fluid is analyzed. The governing equations are given by one-dimensional mass, momentum and constitutive equations which arise in the slender body approximation by cross-sectional averaging of the two-dimensional axisymmetric Stokes equations with free boundary. Existence, uniqueness and regularity results are proved by means of fixed point arguments, energy estimates and weak/weak* convergence methods. The difficulty in this problem lies with the constitutive model of the Maxwell fluid: when both the outflow velocity at the spinneret and the pulling velocity at take up are prescribed, a boundary condition can be imposed for only one of the two elastic stress components at the inlet. The absence of the second stress boundary condition makes the mathematical analysis of the problem hard.
\end{abstract}

Key words and phrases: nonlinear viscoelasticity; UCM fluid; inlet stress conditions; existence, uniqueness and regularity of solutions; compatibility conditions; energy estimates; Schauder fixed point theorem

\section{Introduction}

In industrial applications such as fiber spinning and film casting polymeric melts and solutions are extruded through dies to form synthetic fibers and films. In these flows the polymeric liquid is withdrawn from a reservoir through a circular orifice (spinneret) or slit die and axially stretched. The resulting thin fiber or film is then wound up and passed on to post-processing.

The rheological properties of the liquid undergoing the extension strongly determine its flow behavior. Various constitutive models derived from microstructural or phenomenological considerations have been studied numerically in the hope to better understand the prevalent flow instabilities and other physical

\footnotetext{
* Corresponding author; electronic mail: thagen@memphis.edu
}

†electronic mail: dkurmshv@memphis.edu 
effects occurring during fiber and film forming flows of actual viscous and viscoelastic liquids. Among these the constitutive theory of the upper-convected Maxwell (UCM) fluid plays a special role since it has a microstructural basis and is the principal representative for a large class of constitutive equations in differential form. Even though its physical correctness and applicability to flows of real liquids is certainly questionable, more realistic fluid models (such as the Phan-Thien-Tanner and Giesekus fluids) can be obtained from the UCM fluid, see [1].

Here we consider isothermal fiber spinning of the upper-convected Maxwell fluid in the case of negligible inertia, gravity and surface tension. All equations are stated in dimensionless form. We denote time by $t$, the axial variable by $z$, the fiber radius by $r=r(t, z)$, and the axial velocity by $v=v(t, z)$. The quantities $S=S(t, z)$ and $T=T(t, z)$ denote the radial and axial elastic stresses, respectively. Then the governing equations consist of the equation for mass conservation

$$
\left(r^{2}\right)_{t}+\left(v r^{2}\right)_{z}=0
$$

the equation for momentum conservation

$$
\left(r^{2}(S-T)\right)_{z}=0,
$$

and the constitutive equations for the upper-convected Maxwell fluid

$$
\begin{aligned}
& \text { We }\left(S_{t}+v S_{z}+S v_{z}\right)+S=-v_{z} \\
& \text { We }\left(T_{t}+v T_{z}-2 T v_{z}\right)+T=2 v_{z}
\end{aligned}
$$

The equations are stated on the normalized domain $0 \leq z \leq 1, t \geq 0$. Here the inlet/spinneret and take-up point are assumed at $z=0$ and $z=1$, respectively. The quantity We is a dimensionless (positive) relaxation time, called Weissenberg number, which is a measure for the elasticity of the fluid. To close the formulation of the problem we impose the boundary conditions

$$
\begin{aligned}
& r(t, 0)=1, \\
& v(t, 0)=1, \\
& v(t, 1)=D>1, \\
& S(t, 0)=0,
\end{aligned}
$$

and initial conditions of the form

$$
\begin{aligned}
& r(0, z)=r^{0}(z), \\
& S(0, z)=S^{0}(z), \\
& T(0, z)=T^{0}(z) .
\end{aligned}
$$

The quantity $D>1$, referred to as "draw ratio," is a dimensionless velocity at the take-up point $z=1$. The governing equations can be shown to arise in the slender body approximation of the axisymmetric Stokes equations with moving 
boundary. In the purely viscous case the governing equations are essentially due to Matovich and Pearson [7]. Further details are given in [11].

The boundary conditions discussed here are the ones considered by Forest and Wang in [2]. They are motivated by the desire to control the outflow and take-up velocities as well as the flow rate at the spinneret. Condition (1.8) is based on the observation that the second normal stress difference of the upperconvected Maxwell fluid vanishes inside the spinneret and that the radial elastic stress is expected to be small compared to the axial stress, at least for large Weissenberg numbers. A discussion of these boundary conditions is given in [2, 10]. We emphasize specifically that imposing an additional boundary condition for the axial elastic stress would render the governing equations overdetermined. This observation will be rigorously shown to follow from the results presented in this work. We also note that there is little mathematical difference in prescribing the radial or axial elastic stress at the inlet or a ratio of the two as long as not both elastic stress components are given. For the sake of presentation we have chosen the boundary values in (1.5)-(1.8) constant. All our results, however, will hold true (with minor modifications) for more general right-hand sides.

As was pointed out in [10], the boundary conditions chosen here are an idealization of the physical reality. There is no consensus on which conditions are physically most appropriate and enforceable in actual spinning applications of viscoelastic fluids. The boundary conditions listed above have, however, been commonly used in the literature.

Several authors have commented on the difficulties present in the governing equations due to the absence of one stress boundary condition, see e.g. [8, 9, 10]. This difficulty becomes apparent when one attempts to solve the governing equations numerically [8, 9]. For viscoelastic fluids with constitutive theory in integral form or for purely viscous flow this problem does not arise: in the former case a stress history condition is imposed $[8,9]$, while in the latter case the stresses are given directly in terms of the velocity gradient.

In this work we address the solvability of the boundary-initial value problem given by Eqs. (1.1)-(1.11). We establish a (local-in-time) existence and uniqueness result of rather smooth solutions in suitably chosen function spaces. Our objective is to show the existence of solutions which allow the interpretation of the governing equations in the sense of classical derivatives. Several analytical results about the equations of fiber spinning were obtained by Hagen [3, 4] and Hagen and Renardy [6]. In these works either viscous stresses were included in the constitutive equations [4], thus allowing both stress boundary conditions at the inlet, or both elastic stress components were given at the inlet and one of the velocity components was dropped $[3,6]$. In either case the difficulty encountered with the absent stress boundary condition here was avoided.

The analytic approach chosen in $[3,6]$ and for the earlier nonisothermal viscous case in [5] was based on a contraction mapping argument in certain Sobolev-Bochner spaces. This approach, although being effective, is somewhat tedious and technically demanding. In [4] the contraction mapping argument was replaced by a compactness result and the Schauder fixed point theorem. In this work we will pursue a similar strategy. In addition we will make use 
of a crucial estimate for solutions of the linear transport equation in a certain regularity class $[3,5]$. This result is included in the following section.

\section{The Linear Transport Equation}

Let $a<b, t^{\prime}>0$ and $m, n \in \mathbb{N}_{0}$. Throughout this work we will use the following abbreviations:

- $\|\cdot\|_{p}$ for the norm on the Lebesgue space $L^{p}(a, b), 1 \leq p \leq \infty$,

- $\|\cdot\|_{H^{n}}$ for the norm on the Sobolev space $H^{n}(a, b)$,

- $\|\cdot\|_{m, n}$ for the norm on the Sobolev-Bochner space $W^{m, \infty}\left(\left[0, t^{\prime}\right] ; H^{n}(a, b)\right)$.

Moreover, for functions $h \in L^{\infty}\left(\left[0, t^{\prime}\right] ; H^{2}(a, b)\right) \cap W^{1, \infty}\left(\left[0, t^{\prime}\right] ; H^{1}(a, b)\right)$ we define the norm ||$|\cdot|||$ by

$$
\left\|\left.|h|\right|^{2}=\right\| h\left\|_{0,2}^{2}+\right\| h \|_{1,1}^{2} .
$$

It will be clear in each situation what the concrete values of $a, b$ and $t^{\prime}$ are.

Definition 2.1 The space $\mathbb{B} \mathbb{R}\left(0, t^{\prime} ; 0,1\right)$ of boundary-regular functions consists of all functions $g=g(t, x)$ on $\left[0, t^{\prime}\right] \times[0,1]$ such that

$$
\begin{aligned}
& g \in W^{1, \infty}\left(\left[0, t^{\prime}\right] ; H^{1}(0,1)\right) \cap L^{\infty}\left(\left[0, t^{\prime}\right] ; H^{2}(0,1)\right), \\
& g_{x}(\cdot, 0), g_{x}(\cdot, 1) \in H^{1}\left(0, t^{\prime}\right) .
\end{aligned}
$$

The space $\mathbb{B} \mathbb{R}\left(0, t^{\prime} ; 0,1\right)$ is endowed with the energy norm

$$
\mathcal{E}(g) \stackrel{\text { def }}{=}\left(\|g\|_{0,2}^{2}+\|g\|_{1,1}^{2}+\left\|g_{x}(\cdot, 0)\right\|_{H^{1}}^{2}+\left\|g_{x}(\cdot, 1)\right\|_{H^{1}}^{2}\right)^{\frac{1}{2}} .
$$

The importance of the notion of "boundary-regularity" lies in the following theorem and its corollary. For details and proofs we refer to $[3,5]$.

Theorem 2.2 Let $f, p, u^{0}$ and $u^{*}$ be functions such that

$$
\begin{aligned}
& p, f \in \mathbb{B} \mathbb{R}\left(0, t^{\prime} ; 0,1\right) \\
& p>0 \text { on }\left[0, t^{\prime}\right] \times[0,1], \\
& u^{0} \in H^{2}(0,1), \\
& u^{*} \in H^{2}\left(0, t^{\prime}\right), \\
& u^{0}(0)=u^{*}(0), \\
& u_{t}^{*}(0)+p(0,0) u_{x}^{0}(0)=f(0,0) .
\end{aligned}
$$

Then the boundary-initial value problem on $\left[0, t^{\prime}\right] \times[0,1]$

$$
\begin{aligned}
& u_{t}(t, x)+p(t, x) u_{x}(t, x)=f(t, x), \\
& u(0, x)=u^{0}(x), \\
& u(t, 0)=u^{*}(t)
\end{aligned}
$$


has a solution $u$ such that

$$
\begin{aligned}
& u \in C^{1}\left(\left[0, t^{\prime}\right] ; H^{1}(0,1)\right) \cap C\left(\left[0, t^{\prime}\right] ; H^{2}(0,1)\right) \cap \mathbb{B} \mathbb{R}\left(0, t^{\prime} ; 0,1\right), \\
& u \text { is unique in } W^{1, \infty}\left(\left[0, t^{\prime}\right] ; L^{2}(0,1)\right) \cap L^{\infty}\left(\left[0, t^{\prime}\right] ; H^{1}(0,1)\right) .
\end{aligned}
$$

The proof of this theorem contains an important estimate, which we state here in a form most useful for the following developments. See Lemma 3.15 and Corollary 3.17 and the corresponding proofs in [5].

Corollary 2.3 For $t_{0}>0$ there exists a polynomial $P$ and a constant $C>0$ such that, for each $0<t^{\prime} \leq t_{0}$ and each solution $u$ of the boundary-initial value problem $(2.10)-(2.12)$ on $\left[0, t^{\prime}\right] \times[0,1]$ with functions $f, p, u^{0}$ and $u^{*}$ satisfying the conditions (2.4)-(2.9), the following holds true:

$$
\begin{aligned}
& \|u\|_{0,2}^{2} \leq P\left(\mu^{-1}\right)\left(\left\|u^{0}\right\|_{H^{2}}^{2}+\left(1+\|p\|_{0,2}^{2}\right)^{3}\left\|u^{*}\right\|_{H^{2}}^{2}+\right. \\
& \left.t^{\prime}(1+\||| p||)^{3}\left(\left\|u^{*}\right\|_{H^{2}}^{2}+\||f|\|^{2}\right)\right) e^{(C\|\| p \mid \|+1) t^{\prime}}, \\
& \mathcal{E}(u)^{2} \leq P\left(\mu^{-1}\right)\left(\left\|u^{0}\right\|_{H^{2}}^{2}+\left\|p(0, \cdot) u_{x}^{0}+f(0, \cdot)\right\|_{H^{1}}^{2}+\left(1+\|p\|_{0,2}^{2}\right)^{3}\left\|u^{*}\right\|_{H^{2}}^{2}+\right. \\
& \left.t^{\prime}(1+\||p|\|)^{3}\left(\left\|u^{*}\right\|_{H^{2}}^{2}+\|\| f \|^{2}\right)+t^{\prime}\|\mid p\|\left\|^{2}\right\| u \|_{0,2}\right) \times \\
& e^{C\left(\|p\| \mid\|+\| u \|_{0,2}+1\right) t^{\prime}}
\end{aligned}
$$

where $\mu$ is the minimum value of $p$ on $\left[0, t^{\prime}\right] \times[0,1]$.

\section{The Compatibility Conditions and the Statement of the Main Result}

In this section we state our principal existence/uniqueness result and set the stage for the proofs in later sections. Compatibility conditions for the boundary/initial values are discussed.

Definition 3.1 A vector field $(r, v, S, T)$, defined on $\left[0, t^{\prime}\right] \times[0,1]$ for some $t^{\prime}>$ 0 , is a solution of the boundary-initial value problem (1.1)-(1.11) if

$$
\begin{aligned}
& r, v, S, T \in C^{1}\left(\left[0, t^{\prime}\right] ; H^{1}(0,1)\right) \cap C\left(\left[0, t^{\prime}\right] ; H^{2}(0,1)\right), \\
& r, v, S, T \text { satisfy Eqs. }(1.1)-(1.4), \\
& r \text { satisfies Eqs. }(1.5),(1.9) \text { and } r>0, \\
& S \text { satisfies Eqs. }(1.8),(1.10), \\
& T \text { satisfies Eq. }(1.11), \\
& v \text { satisfies Eqs. }(1.6),(1.7) \text { and } v>0 .
\end{aligned}
$$

The velocity and radius are required to be positive for physical reasons. Observe that the regularity of the solution is strong enough to guarantee continuous 
differentiability on $\left[0, t^{\prime}\right] \times[0,1]$. We tacitly assume that the boundary and initial data are regular enough (the regularity requirements will be made clear later on).

The smoothness required for solutions can only hold true for initial and boundary data that satisfy certain compatibility conditions that are in agreement with Eqs. (1.1)-(1.11). Specifically, for consistency we need

$$
r^{0}(z)>0 \text { for } 0 \leq z \leq 1
$$

and

$$
\left(r^{0}(z)\right)^{2}\left(S^{0}(z)-T^{0}(z)\right)=\text { const. }
$$

However, regularity of solution also requires that the boundary/initial data and their respective derivatives satisfy additional conditions. In particular, to match initial and boundary data at $t=0, z=0$, we impose the constraints

$$
r^{0}(0)=1 \quad \text { and } \quad S^{0}(0)=0 .
$$

To match first derivatives, we demand

$$
r_{z}^{0}(0)=-\frac{1}{2} v_{z}(0,0) \quad \text { and } \quad S_{z}^{0}(0)=-\frac{v_{z}(0,0)}{\mathrm{We}},
$$

where Eqs. (1.1)-(1.4) and (1.6)-(1.7) give

$$
v(t, z)=D+\int_{1}^{z} \frac{T(t, 0)+\mathrm{We} T_{t}(t, 0)}{-2 \mathrm{We} T(t, 0)+3(r(t, x))^{2}(1+\mathrm{We} T(t, x))} d x
$$

and

$$
v_{z}(0,0)=\frac{T^{0}(0)+\mathrm{We} T_{t}(0,0)}{3+\mathrm{We} T^{0}(0)}
$$

Notice that the dependence of the velocity on the unknown boundary data of $T$ is the core problem that we will have to address. To this end, we introduce the unknown boundary function

$$
X(t)=T(t, 0)
$$

and note that Eq. (3.11), evaluated at $z=0$, gives a first-order ordinary differential equation for $X$

$$
\text { We } X_{t}(t)+X(t)=\frac{D-1}{\int_{0}^{1} \frac{1}{-2 \mathrm{We} X(t)+3(r(t, x))^{2}(1+\mathrm{We} T(t, x))} d x} .
$$

Together with the initial condition

$$
X(0)=T^{0}(0)
$$

this equation is in principle solvable when all the other quantities are known and division by zero is avoided. Observe that $X$ solving Eq. (3.14) implies that 
$v=1$ at $z=0$ in Eq. (3.11). For such a solution $X$ we still must make sure that its first derivative is correctly related to the initial value $T^{0}$. First, Eq. (3.14) imposes the condition

$$
X_{t}(0)=-\frac{1}{\mathrm{We}} T^{0}(0)+\frac{D-1}{\int_{0}^{1} \frac{\mathrm{We}}{-2 \mathrm{We} T^{0}(0)+3\left(r^{0}(x)\right)^{2}\left(1+\mathrm{We} T^{0}(x)\right)} d x} .
$$

Second, Eq. (1.4) requires

$$
X_{t}(0)=-T_{z}^{0}(0)+\left(2 v_{z}(0,0)-\frac{1}{\mathrm{We}}\right) T^{0}(0)+\frac{2}{\mathrm{We}} v_{z}(0,0)
$$

Using Eq. (3.12) in (3.17), we obtain

$$
X_{t}(0)=-\frac{1}{\mathrm{We}} T^{0}(0)+\frac{\mathrm{We} T^{0}(0)+3}{\mathrm{We} T^{0}(0)-1} T_{z}^{0}(0) .
$$

Hence Eqs. (3.16), (3.18) give the condition

$$
\frac{D-1}{\int_{0}^{1} \frac{\mathrm{We}}{-2 \mathrm{We} T^{0}(0)+3\left(r^{0}(x)\right)^{2}\left(1+\mathrm{We}^{0}(x)\right)} d x}=\frac{\operatorname{We} T^{0}(0)+3}{\operatorname{We} T^{0}(0)-1} T_{z}^{0}(0) .
$$

In summary, in addition to the compatibility conditions (3.7)-(3.9) and (3.19), we have Eqs. (3.10) which assume the form

$$
\begin{aligned}
& r_{z}^{0}(0)=-\frac{1}{2} \frac{\mathrm{We}}{\mathrm{We} T^{0}(0)-1} T_{z}^{0}(0), \\
& S_{z}^{0}(0)=-\frac{1}{\operatorname{We} T^{0}(0)-1} T_{z}^{0}(0) .
\end{aligned}
$$

Here we have used the identity

$$
v_{z}(0,0)=\frac{\mathrm{We}}{\operatorname{We} T^{0}(0)-1} T_{z}^{0}(0) .
$$

Since the velocity has to be positive initially, we demand that for $0 \leq z \leq 1$

$$
\begin{aligned}
& v(0, z)=v^{0}(z)=D+ \\
& \quad \int_{1}^{z} \frac{\mathrm{We}\left(\mathrm{We} T^{0}(0)+3\right) T_{z}^{0}(0)}{\left(\mathrm{We} T^{0}(0)-1\right)\left(3\left(r^{0}(x)\right)^{2}\left(1+\mathrm{We} T^{0}(x)\right)-2 \mathrm{We} T^{0}(0)\right)} d x>0 .
\end{aligned}
$$

Of course, it is clear that all expressions appearing in denominators have to be nonzero and that the velocity cannot be constant. Hence we also have to ensure that

$$
\begin{aligned}
& T^{0}(0) \notin\left\{-\frac{3}{\mathrm{We}}, \frac{1}{\mathrm{We}}\right\}, \\
& T_{z}^{0}(0) \neq 0, \\
& 3\left(r^{0}(z)\right)^{2}\left(1+\mathrm{We}^{0}(z)\right)-2 \mathrm{We} T^{0}(0) \neq 0, \quad 0 \leq z \leq 1 .
\end{aligned}
$$


To demonstrate that the set of initial conditions satisfying the compatibility requirements above is nonempty, we give one mathematically possible choice of data.

Example 3.2 Let $m=\frac{1}{4} \ln (2 D-1)$ and set

$$
r^{0}(z)=e^{-m z}, \quad S^{0}(z)=-\frac{1}{\mathrm{We}}+\frac{1}{\mathrm{We}} e^{-2 m z}=T^{0}(z)
$$

With these functions it follows readily that conditions (3.7)-(3.9), (3.19)-(3.21), (3.23)-(3.26) hold true.

Throughout the remainder of this work we will tacitly assume that all initial data considered satisfy the compatibility conditions (3.7)-(3.9), (3.19)-(3.21), (3.23)-(3.26).

We are now in a position to state the central result of this work.

Theorem 3.3 Suppose the initial values $r^{0}, S^{0}, T^{0}$ are given in $H^{2}(0,1)$ (and are compatible). Then the boundary-initial value problem (1.1)-(1.11) has a solution $(r, v, S, T)$ on $\left[0, t^{\prime}\right] \times[0,1]$ for some $t^{\prime}>0$. This solution has the properties

$$
\begin{aligned}
& r, S, T \in \bigcap_{k=0}^{2} C^{k}\left(\left[0, t^{\prime}\right] ; H^{2-k}(0,1)\right), \\
& r, S, T \in \mathbb{B} \mathbb{R}\left(0, t^{\prime} ; 0,1\right), \\
& v \in \bigcap_{k=0}^{2} C^{k}\left(\left[0, t^{\prime}\right] ; H^{3-k}(0,1)\right) .
\end{aligned}
$$

Moreover, $(r, v, S, T)$ is the unique solution of the boundary-initial value problem (1.1)-(1.11) in

$$
\left(\mathbb{B R}\left(0, t^{\prime} ; 0,1\right)\right)^{4}
$$

The proof of Theorem 3.3 will be split up in several steps. The idea is to use the Schauder fixed point theorem on a suitable compact set. A similar strategy was applied in the much simpler case of the Jeffreys fluid in [4] where both elastic stress components were prescribed at the inlet.

\section{The Set of Potential Solutions}

We begin by introducing the set in which we will search for a solution.

Definition 4.1 For $L, M, t^{\prime}>0$, let $\mathbb{S}\left(t^{\prime}, L, M\right)$ be the set of all functions 
$(q, U, V, Y)$ such that

$$
\begin{aligned}
& q, U, V \in \mathbb{B} \mathbb{R}\left(0, t^{\prime} ; 0,1\right) \quad \text { and } Y \in H^{2}\left(0, t^{\prime}\right) \\
& \mathcal{E}(q)^{2}+\mathcal{E}(U)^{2}+\mathcal{E}(V)^{2} \leq L^{2} \quad \text { and } \quad\|Y\|_{H^{2}} \leq M \\
& q(0, z)=r^{0}(z) \quad \text { and } \quad q(t, 0)=1 \\
& U(0, z)=S^{0}(z) \quad \text { and } \quad U(t, 0)=0 \\
& V(0, z)=T^{0}(z) \\
& Y(0)=T^{0}(0)
\end{aligned}
$$

If the constants $L$ and $M$ are sufficiently large, then $\left(r^{0}, S^{0}, T^{0}, T^{0}(0)\right)$ belongs to the set $\mathbb{S}\left(t^{\prime}, L, M\right)$ for any $t^{\prime}>0$. Also, by construction of $\mathbb{S}\left(t^{\prime}, L, M\right)$, we can find constants $C=C(L)>0$ and $c=c(M)>0$ such that

$$
\begin{aligned}
& \left|q(t, z)-r^{0}(z)\right| \leq \int_{0}^{t}\left|q_{t}(s, z)\right| d s \leq C t^{\prime} \\
& \left.\left|V(t, z)-T^{0}(z)\right| \leq \int_{0}^{t} \mid V_{t}(s, z)\right) \mid d s \leq C t^{\prime} \\
& \left|Y(t)-T^{0}(0)\right| \leq \int_{0}^{t}\left|Y_{t}(s)\right| d s \leq c \sqrt{t^{\prime}}
\end{aligned}
$$

for all $(q, U, V, Y)$ in $\mathbb{S}\left(t^{\prime}, L, M\right)$. These estimates follow from the observation that $q_{t}, V_{t} \in L^{\infty}\left(\left(0, t^{\prime}\right) ; H^{1}(0,1)\right)$ and $Y_{t} \in H^{1}\left(0, t^{\prime}\right)$ with norms bounded in terms of $L$ and $M$, respectively. Since condition (3.26) is assumed to hold, we obtain the following result.

Proposition 4.2 There exist $L, M>0$ such that the set $\mathbb{S}(t, L, M)$ is nonempty for all $t>0$. Moreover, if $\mathbb{S}\left(t_{0}, L, M\right) \neq \emptyset$, then there is $0<t^{*} \leq t_{0}$ such that, for any $0<t^{\prime} \leq t^{*}$ and $(q, U, V, Y) \in \mathbb{S}\left(t^{\prime}, L, M\right)$,

$$
3(q(t, z))^{2}(1+\mathrm{We} V(t, z))-2 \mathrm{We} Y(t) \neq 0 \quad \text { for } 0 \leq t \leq t^{\prime}, 0 \leq z \leq 1 .
$$

From now on we will tacitly assume that the conclusions of Proposition 4.2 hold true for our choices of $t^{\prime}, L, M>0$.

Theorem 4.3 $\mathbb{S}\left(t^{\prime}, L, M\right)$ is convex and compact in $\left(L^{2}\left(\left(0, t^{\prime}\right) \times(0,1)\right)\right)^{3} \times$ $L^{2}\left(0, t^{\prime}\right)$.

Proof. $\mathbb{S}\left(t^{\prime}, L, M\right)$ is convex by construction. It is precompact in the space $\left(L^{2}\left(\left(0, t^{\prime}\right) \times(0,1)\right)\right)^{3} \times L^{2}\left(0, t^{\prime}\right)$ since it is bounded in $\left(H^{1}\left(\left(0, t^{\prime}\right) \times(0,1)\right)\right)^{3} \times$ $H^{1}\left(0, t^{\prime}\right)$, the latter space being compactly embedded in the former. Suppose $\left(q_{n}, U_{n}, V_{n}, Y_{n}\right)_{n \in \mathbb{N}}$ is a Cauchy sequence in $\left(L^{2}\left(\left(0, t^{\prime}\right) \times(0,1)\right)\right)^{3} \times L^{2}\left(0, t^{\prime}\right)$ with limit $\left(q_{0}, U_{0}, V_{0}, Y_{0}\right)$ such that

$$
\left(q_{n}, U_{n}, V_{n}, Y_{n}\right) \in \mathbb{S}\left(t^{\prime}, L, M\right) \text { for all } n \in \mathbb{N} .
$$


We will show that $\left(q_{0}, U_{0}, V_{0}, Y_{0}\right)$ belongs to $\mathbb{S}\left(t^{\prime}, L, M\right)$. First, since $\left(Y_{n}\right)_{n}$ is bounded in $H^{2}\left(0, t^{\prime}\right),\left(Y_{n}\right)_{n}$ has a weakly convergent subsequence with weak limit $Y^{*}$ in $H^{2}\left(0, t^{\prime}\right)$. Since weak convergence in $H^{2}\left(0, t^{\prime}\right)$ implies strong convergence in $L^{2}\left(0, t^{\prime}\right)$, we conclude $Y^{*}=Y_{0}$, hence $Y_{0} \in H^{2}\left(0, t^{\prime}\right)$ and $\left\|Y_{0}\right\|_{H^{2}} \leq M$.

Next let $\left(Z_{n}\right)_{n}$ be one of the sequences $\left(q_{n}\right)_{n},\left(U_{n}\right)_{n}$ or $\left(V_{n}\right)_{n}$ and denote its limit in $L^{2}\left(\left(0, t^{\prime}\right) \times(0,1)\right)$ by $Z_{0}$. Since $\left(Z_{n}\right)_{n}$ is bounded in $L^{\infty}\left(\left[0, t^{\prime}\right] ; H^{2}(0,1)\right)$ and since $L^{\infty}\left(\left[0, t^{\prime}\right] ; H^{2}(0,1)\right)$ is the conjugate of the separable Banach space $L^{1}\left(\left[0, t^{\prime}\right] ; H^{2}(0,1)\right)$, we can extract a weak ${ }^{*}$ convergent subsequence with weak ${ }^{*}$ limit $Z^{*}$ in $L^{\infty}\left(\left[0, t^{\prime}\right] ; H^{2}(0,1)\right)$. However, this subsequence converges weakly in $L^{2}\left(\left(0, t^{\prime}\right) \times(0,1)\right)$ with weak limit $Z^{*}$. Since the subsequence also converges strongly in $L^{2}\left(\left(0, t^{\prime}\right) \times(0,1)\right)$ to $Z_{0}$, we have $Z^{*}=Z_{0}$, hence $Z_{0}$ belongs to $L^{\infty}\left(\left[0, t^{\prime}\right] ; H^{2}(0,1)\right)$.

Since $\left(Z_{n}\right)_{n}$ is also bounded in $W^{1, \infty}\left(\left[0, t^{\prime}\right] ; H^{1}(0,1)\right)$ and thus in $H^{1}\left(\left(0, t^{\prime}\right) \times\right.$ $(0,1))$, we can extract yet another subsequence - call it $\left(Z_{n}\right)_{n}$ for simplicity such that

$$
\begin{aligned}
& \left(\frac{\partial}{\partial t} Z_{n}\right)_{n} \text { converges weak }{ }^{*} \text { in } L^{\infty}\left(\left[0, t^{\prime}\right] ; H^{1}(0,1)\right) \text { with weak }{ }^{*} \text { limit } z^{*} \\
& \left(Z_{n}\right)_{n} \text { converges weakly in } H^{1}\left(\left(0, t^{\prime}\right) \times(0,1)\right) .
\end{aligned}
$$

Since weak convergence in $H^{1}\left(\left(0, t^{\prime}\right) \times(0,1)\right)$ implies strong convergence in $L^{2}\left(\left(0, t^{\prime}\right) \times(0,1)\right)$, we conclude that that the weak limit of $\left(Z_{n}\right)_{n}$ in $H^{1}\left(\left(0, t^{\prime}\right) \times\right.$ $(0,1))$ is $Z_{0}$. Thus $Z_{0}$ belongs to $H^{1}\left(\left(0, t^{\prime}\right) \times(0,1)\right)$. Moreover, the sequence $\left(\frac{\partial}{\partial t} Z_{n}\right)_{n}$ converges weakly to $\frac{\partial}{\partial t} Z_{0}$ in $L^{2}\left(\left[0, t^{\prime}\right] ; H^{1}(0,1)\right)$. Since the sequence of time derivatives converges weak ${ }^{*}$ to $z^{*}$ in $L^{\infty}\left(\left[0, t^{\prime}\right] ; H^{1}(0,1)\right)$ and thus weakly in $L^{2}\left(\left[0, t^{\prime}\right] ; H^{1}(0,1)\right)$, we have $z^{*}=\frac{\partial}{\partial t} Z_{0}$. This proves that $Z_{0}$ belongs to $W^{1, \infty}\left(\left[0, t^{\prime}\right] ; H^{1}(0,1)\right)$ as well.

Next we note that the sequences $\left(\frac{\partial}{\partial x} Z_{n}(\cdot, 0)\right)_{n}$ and $\left(\frac{\partial}{\partial x} Z_{n}(\cdot, 1)\right)_{n}$ are bounded in $H^{1}\left(0, t^{\prime}\right)$. Hence we may pass again to a subsequence of $\left(Z_{n}\right)_{n}$, called $\left(Z_{n}\right)_{n}$ as well, such that $\left(\frac{\partial}{\partial x} Z_{n}(\cdot, 0)\right)_{n}$ and $\left(\frac{\partial}{\partial x} Z_{n}(\cdot, 1)\right)_{n}$ converge weakly in $H^{1}\left(0, t^{\prime}\right)$. Since weak convergence in $H^{1}\left(0, t^{\prime}\right)$ implies strong convergence in $L^{2}\left(0, t^{\prime}\right)$, we deduce that the sequences have the strong limits $\frac{\partial}{\partial x} Z_{0}(\cdot, 0)$ and $\frac{\partial}{\partial x} Z_{0}(\cdot, 1)$ in $L^{2}\left(0, t^{\prime}\right)$, respectively, and that $\frac{\partial}{\partial x} Z_{0}(\cdot, 0)$ and $\frac{\partial}{\partial x} Z_{0}(\cdot, 1)$ belong to $H^{1}\left(0, t^{\prime}\right)$.

Finally, it is clear that $Z_{0}$ satisfies the relevant initial and boundary conditions (if applicable) and that

$$
\mathcal{E}\left(q_{0}\right)^{2}+\mathcal{E}\left(U_{0}\right)^{2}+\mathcal{E}\left(V_{0}\right)^{2} \leq L^{2}
$$

the latter property following from standard norm estimates of weakly and weak* convergent sequences. In summary we conclude that the set $\mathbb{S}\left(t^{\prime}, L, M\right)$ is precompact and closed in $\left(L^{2}\left(\left(0, t^{\prime}\right) \times(0,1)\right)\right)^{3} \times L^{2}\left(0, t^{\prime}\right)$, therefore compact. 


\section{The Schauder Map}

In this section we construct a map $\Sigma$ on a suitable set $\mathbb{S}\left(t^{\prime}, L, M\right)$ and show that the Schauder fixed point theorem applies.

For $(q, U, V, Y) \in \mathbb{S}\left(t^{\prime}, L, M\right)$ we define the operators $w$ and $w^{\prime}$ by

$$
w(q, V, Y)(t, z)=D+\frac{\int_{1}^{z} \frac{(D-1) d x}{-2 \mathrm{We} Y(t)+3(q(t, x))^{2}(1+\operatorname{We} V(t, x))}}{\int_{0}^{1} \frac{d x}{-2 \mathrm{We} Y(t)+3(q(t, x))^{2}(1+\operatorname{We} V(t, x))}}
$$

and

$$
w^{\prime}(q, V, Y)(t, z)=\frac{\frac{(D-1)}{-2 \mathrm{We} Y(t)+3(q(t, z))^{2}(1+\mathrm{We} V(t, z))}}{\int_{0}^{1} \frac{d x}{-2 \mathrm{We} Y(t)+3(q(t, x))^{2}(1+\mathrm{We} V(t, x))}} .
$$

By Proposition 4.2, $w(q, V, Y)$ and $w^{\prime}(q, V, Y)$ are well-defined. Moreover, both $w(q, V, Y)$ and $w^{\prime}(q, V, Y)$ are boundary-regular functions and $w(q, V, Y)$ takes the minimum value 1 . Next we let $\tilde{q}, \tilde{U}$ and $\tilde{V}$ be the solutions of the following boundary-initial value problems on $\left[0, t^{\prime}\right] \times[0,1]$

$$
\begin{aligned}
& \tilde{q}_{t}+w(q, V, Y) \tilde{q}_{z}=-\frac{1}{2} w^{\prime}(q, V, Y) q, \\
& \tilde{q}(t, 0)=1, \quad \tilde{q}(0, z)=r^{0}(z), \\
& \tilde{U}_{t}+w(q, V, Y) \tilde{U}_{z}=-\left(w^{\prime}(q, V, Y)+\frac{1}{\mathrm{We}}\right) U-\frac{1}{\mathrm{We}} w^{\prime}(q, V, Y), \\
& \tilde{U}(t, 0)=0, \quad \tilde{U}(0, z)=S^{0}(z), \\
& \tilde{V}_{t}+w(q, V, Y) \tilde{V}_{z}=\left(2 w^{\prime}(q, V, Y)-\frac{1}{\mathrm{We}}\right) V+\frac{2}{\mathrm{We}} w^{\prime}(q, V, Y), \\
& \tilde{V}(t, 0)=Y(t), \quad \tilde{V}(0, z)=T^{0}(z) .
\end{aligned}
$$

Since the data are assumed compatible in the sense of Section 3 and the coefficients have the required regularity, Theorem 2.2 applies. Hence $\tilde{q}, \tilde{U}$ and $\tilde{V}$ are well-defined and belong to

$$
C^{1}\left(\left[0, t^{\prime}\right] ; H^{1}(0,1)\right) \cap C\left(\left[0, t^{\prime}\right] ; H^{2}(0,1)\right) \cap \mathbb{B} \mathbb{R}\left(0, t^{\prime} ; 0,1\right) .
$$

Finally we let $\tilde{Y}$ be the solution of the initial value problem

$$
\begin{aligned}
& \tilde{Y}_{t}+\frac{1}{\mathrm{We}} \tilde{Y}=\frac{D-1}{\mathrm{We}} \frac{1}{\int_{0}^{1} \frac{d x}{-2 \mathrm{We} Y(t)+3(q(t, x))^{2}(1+\mathrm{We} V(t, x))}} \\
& \tilde{Y}(0)=T^{0}(0) .
\end{aligned}
$$


The regularity of the right-hand side in Eq. (5.10) proves that

$$
\tilde{Y} \in H^{2}\left(0, t^{\prime}\right)
$$

Note that for $\tilde{Y}$ given by $(5.10)-(5.11)$

$$
w(q, V, Y)(t, z)=D+\int_{1}^{z} \frac{\tilde{Y}(t)+\mathrm{We} \tilde{Y}_{t}(t)}{-2 \mathrm{We} Y(t)+3(q(t, x))^{2}(1+\mathrm{We} V(t, x))} d x
$$

The Schauder map $\Sigma$ is now defined by

$$
\Sigma:\left\{\begin{array}{ccc}
\mathbb{S}\left(t^{\prime}, L, M\right) & \longrightarrow & L^{2}\left(\left(0, t^{\prime}\right) \times(0,1)\right)^{3} \times L^{2}\left(0, t^{\prime}\right) \\
(q, U, V, Y) & \longmapsto & (\tilde{q}, \tilde{U}, \tilde{V}, \tilde{Y})
\end{array}\right.
$$

As noted above, we have the following conclusion.

Proposition 5.1 The operator $\Sigma$ is well-defined on $\mathbb{S}\left(t^{\prime}, L, M\right)$.

Lemma 5.2 There are $L>0, M>0$ and $t^{\prime}>0$ such that the operator $\Sigma$ maps $\mathbb{S}\left(t^{\prime}, L, M\right)$ into $\mathbb{S}\left(t^{\prime}, L, M\right)$.

Proof. Throughout we may assume that $t^{\prime} \leq 1$. We will make use of the estimate (2.16), applied to each of the boundary-initial value problems (5.3)(5.4), (5.5)-(5.6) and (5.7)-(5.8). First for arbitrary $(q, U, V, Y) \in \mathbb{S}\left(t^{\prime}, L, M\right)$, we use an argument along the lines of (4.7)-(4.9) to note that

$$
\begin{aligned}
& \mid 3(q(t, z))^{2}(1+\operatorname{We} V(t, z))-2 \operatorname{We} Y(t)-3\left(r^{0}(z)\right)^{2}\left(1+\operatorname{We} T^{0}(z)\right)+ \\
& \quad 2 \operatorname{We} T^{0}(0) \mid \leq C(L, M) \sqrt{t^{\prime}}
\end{aligned}
$$

for some constant $C(L, M)$ which depends on $L$ and $M$ only (here we have used $\left.t^{\prime} \leq 1\right)$. Hence we may assume that $t^{\prime}$ is chosen small compared to $C(L, M)$ such that, for $0 \leq t \leq t^{\prime}, 0 \leq z \leq 1$

$$
\begin{gathered}
\mid 3(q(t, z))^{2}(1+\operatorname{We} V(t, z))-2 \operatorname{We} Y(t)-3\left(r^{0}(z)\right)^{2}\left(1+\operatorname{We} T^{0}(z)\right)+ \\
2 \operatorname{We}^{0}(0)\left|\leq \frac{1}{2}\right| 3\left(r^{0}(z)\right)^{2}\left(1+\mathrm{We} T^{0}(z)\right)-2 \operatorname{We} T^{0}(0) \mid
\end{gathered}
$$

Hence we can bound the term

$$
\left|-2 \mathrm{We} Y(t)+3(q(t, z))^{2}(1+\mathrm{We} V(t, z))\right|,
$$

its inverse and integrals thereof both above and below in terms of the initial data. Therefore the only term left to consider when taking the first and second derivatives of $w(q, V, Y)$ with respect to $z$ is of the form

$$
6 q q_{z}(1+\mathrm{We} V)+3 q^{2} \mathrm{We} V_{z} .
$$


Here all terms can be estimated by an expression in $L$. However, it is advantageous to note that the regularity properties of $q$ and $V$ imply that there exists a constant $c(L)$, depending on $L$, such that

$$
\begin{aligned}
& \int_{0}^{1}\left(6 q q_{z}(1+\mathrm{We} V)+3 q^{2} \text { We } V_{z}-6 r^{0} r_{z}^{0}\left(1+\mathrm{We} T^{0}\right)-\right. \\
& \left.\quad 3\left(r^{0}\right)^{2} \operatorname{We} T_{z}^{0}\right)^{2} d z \leq c(L) t^{\prime} .
\end{aligned}
$$

It follows that $\|w(q, V, Y)\|_{0,2}$ can be estimated by the initial data up to a term involving $L$ that can be made arbitrarily small if $t^{\prime}$ is chosen sufficiently small.

In order to apply the estimate (2.16) of Corollary 2.3, we note that almost all terms on the right of (2.16) involve only initial data or terms which are bounded by expressions in $L$ and $M$ and multiplied by $t^{\prime}$. Terms of the latter form can therefore be made small or in case of the exponential close to 1 . Only the polynomial involving the minimum value $\mu$ of the flux coefficient $p$ and the third term in the parenthesis on the right of estimate (2.16) are left to be discussed. In the situation here we have $p=w(q, V, Y)$. The polynomial term is trivial since the relevant minimum value $\mu$ assumed by $w(q, V, Y)$ is 1 . The third term involves, however, the boundary data and $\|w(q, V, Y)\|_{0,2}$. As seen above the latter quantity can be bounded in terms of the initial data plus a term multiplied by $t^{\prime}$. Therefore after having taken $L$ and $M$ sufficiently large to accommodate initial and boundary data, we can make $t^{\prime}$ so small in estimate (2.16) that

$$
\mathcal{E}(\tilde{q})^{2}+\mathcal{E}(\tilde{U})^{2}+\mathcal{E}(\tilde{V})^{2} \leq L^{2} .
$$

It remains to estimate the solution of the initial value problem (5.10)-(5.11). To this end we note that

$$
\tilde{Y}(t)=e^{-\frac{t}{\mathrm{We}}} T^{0}(0)+\int_{0}^{t} \frac{(D-1) e^{\frac{s-t}{\mathrm{We}}}}{\int_{0}^{1} \frac{\mathrm{We} d x}{-2 \mathrm{We} Y(s)+3(q(s, x))^{2}(1+\operatorname{We} V(s, x))}} d s .
$$

Hence using the estimate (5.16), $|\tilde{Y}|$ can be bounded in terms of the initial data. Because of Eq. (5.10) the same applies to $\left|\tilde{Y}_{t}\right|$. As we differentiate Eq. (5.10) with respect to $t$, we note that quantities like $|q|,\left|q_{t}\right|,|V|$ and $\left|V_{t}\right|$ are bounded in terms of $L$. Consequently, $\int_{0}^{t^{\prime}} \tilde{Y}_{t t}^{2} d t$ can be estimated by the initial data and an expression of the form $c(L) t^{\prime}$, where $c(L)$ is a constant depending on $L$. However, this result implies that for $M$ chosen large enough to take care of the initial data, $t^{\prime}$ can be taken small enough to enforce

$$
\|\tilde{Y}\|_{H^{2}} \leq M
$$

This concludes the proof.

From now on we may assume that $\Sigma$ maps into $\mathbb{S}\left(t^{\prime}, L, M\right)$. 
Lemma 5.3 The operator $\Sigma$ is continuous on $\mathbb{S}\left(t^{\prime}, L, M\right)$ with respect to the topology of $\left(L^{2}\left(\left[0, t^{\prime}\right] \times[0,1]\right)^{3} \times L^{2}\left(0, t^{\prime}\right)\right.$.

Proof. Let $(q, U, V, Y)$ and $(\bar{q}, \bar{U}, \bar{V}, \bar{Y})$ be in $\mathbb{S}\left(t^{\prime}, L, M\right)$ and set

$$
\begin{aligned}
& (\kappa, \Omega, \Phi, \Psi)=\Sigma(q, U, V, Y), \\
& (\bar{\kappa}, \bar{\Omega}, \bar{\Phi}, \bar{\Psi})=\Sigma(\bar{q}, \bar{U}, \bar{V}, \bar{Y}) .
\end{aligned}
$$

In the following we let $C$ be a generic constant which is allowed to depend on $L, M, t^{\prime}$. First we notice that the regularity of functions in $\mathbb{S}\left(t^{\prime}, L, M\right)$ and some elementary calculations imply that

$$
\begin{aligned}
& \|w(q, V, Y)(t, \cdot)-w(\bar{q}, \bar{V}, \bar{Y})(t, \cdot)\|_{2}^{2} \leq C\left(\|q(t, \cdot)-\bar{q}(t, \cdot)\|_{2}^{2}+\right. \\
& \left.\quad\|V(t, \cdot)-\bar{V}(t, \cdot)\|_{2}^{2}+(Y(t)-\bar{Y}(t))^{2}\right), \\
& \left\|w^{\prime}(q, V, Y)(t, \cdot)-w^{\prime}(\bar{q}, \bar{V}, \bar{Y})(t, \cdot)\right\|_{2}^{2} \leq C\left(\|q(t, \cdot)-\bar{q}(t, \cdot)\|_{2}^{2}+\right. \\
& \left.\quad\|V(t, \cdot)-\bar{V}(t, \cdot)\|_{2}^{2}+(Y(t)-\bar{Y}(t))^{2}\right) .
\end{aligned}
$$

Consequently, as we take the differences of the equations corresponding to Eqs. (5.3)-(5.4) for $\kappa$ and $\bar{\kappa}$, multiply by $\kappa-\bar{\kappa}$ and integrate in $z$ from 0 to 1 , we obtain an estimate of the form

$$
\begin{aligned}
& \frac{d}{d t}\|\kappa(t, \cdot)-\bar{\kappa}(t, \cdot)\|_{2}^{2} \leq C\left(\|\kappa(t, \cdot)-\bar{\kappa}(t, \cdot)\|_{2}^{2}+\|q(t, \cdot)-\bar{q}(t, \cdot)\|_{2}^{2}+\right. \\
& \left.\quad\|V(t, \cdot)-\bar{V}(t, \cdot)\|_{2}^{2}+(Y(t)-\bar{Y}(t))^{2}\right) .
\end{aligned}
$$

Here we have made use of the fact that $w(q, V, Y)$ and $w(\bar{q}, \bar{V}, \bar{Y})$ are positive and that $w(q, V, Y), w(\bar{q}, \bar{V}, \bar{Y}), w^{\prime}(q, V, Y)$ and $w^{\prime}(\bar{q}, \bar{V}, \bar{Y})$ are bounded in terms of $L, M$ and $t^{\prime}$. In an analogous way we obtain

$$
\begin{gathered}
\frac{d}{d t}\|\Omega(t, \cdot)-\bar{\Omega}(t, \cdot)\|_{2}^{2} \leq C\left(\|\Omega(t, \cdot)-\bar{\Omega}(t, \cdot)\|_{2}^{2}+\|q(t, \cdot)-\bar{q}(t, \cdot)\|_{2}^{2}+\right. \\
\left.\|U(t, \cdot)-\bar{U}(t, \cdot)\|_{2}^{2}+\|V(t, \cdot)-\bar{V}(t, \cdot)\|_{2}^{2}+(Y(t)-\bar{Y}(t))^{2}\right) .
\end{gathered}
$$

The corresponding calculation for $\Phi-\bar{\Phi}$ is slightly different because $\Phi-\bar{\Phi}=$ $Y-\bar{Y}$ at $z=0$. (For $\kappa-\bar{\kappa}$ and $\Omega-\bar{\Omega}$ the equivalent boundary term vanishes.) However, the resulting estimate is still of the same form as before

$$
\begin{aligned}
& \frac{d}{d t}\|\Phi(t, \cdot)-\bar{\Phi}(t, \cdot)\|_{2}^{2} \leq C\left(\|\Phi(t, \cdot)-\bar{\Phi}(t, \cdot)\|_{2}^{2}+\|q(t, \cdot)-\bar{q}(t, \cdot)\|_{2}^{2}+\right. \\
& \left.\quad\|V(t, \cdot)-\bar{V}(t, \cdot)\|_{2}^{2}+(Y(t)-\bar{Y}(t))^{2}\right) .
\end{aligned}
$$

Finally, as we take differences of the equations determining $\Psi$ and $\bar{\Psi}$ and multiply by $\Psi-\bar{\Psi}$, we obtain the estimate

$$
\begin{aligned}
& \frac{d}{d t}(\Psi(t)-\bar{\Psi}(t))^{2} \leq C\left((\Psi(t)-\bar{\Psi}(t))^{2}+\|q(t, \cdot)-\bar{q}(t, \cdot)\|_{2}^{2}+\right. \\
& \left.\quad\|V(t, \cdot)-\bar{V}(t, \cdot)\|_{2}^{2}+(Y(t)-\bar{Y}(t))^{2}\right) .
\end{aligned}
$$


We set

$$
\begin{aligned}
& \rho(t)=\|\kappa(t, \cdot)-\bar{\kappa}(t, \cdot)\|_{2}^{2}+\|\Omega(t, \cdot)-\bar{\Omega}(t, \cdot)\|_{2}^{2}+\|\Phi(t, \cdot)-\bar{\Phi}(t, \cdot)\|_{2}^{2}+ \\
& (\Psi(t)-\bar{\Psi}(t))^{2} \\
& \sigma(t)=\|q(t, \cdot)-\bar{q}(t, \cdot)\|_{2}^{2}+\|U(t, \cdot)-\bar{U}(t, \cdot)\|_{2}^{2}+\|V(t, \cdot)-\bar{V}(t, \cdot)\|_{2}^{2}+ \\
& \quad(Y(t)-\bar{Y}(t))^{2}
\end{aligned}
$$

Then estimates (5.27)-(5.30) can be combined to read

$$
\frac{d}{d t} \rho(t) \leq C(\rho(t)+\sigma(t))
$$

Finally Grönwall's inequality gives

$$
\rho(t) \leq C \int_{0}^{t} e^{C(t-s)} \sigma(s) d s .
$$

This implies the claim.

Suppose the map $\Sigma$ has two fixed points, say $(q, U, V, Y)$ and $(\bar{q}, \bar{U}, \bar{V}, \bar{Y})$ in $\mathbb{S}\left(t^{\prime}, L, M\right)$. Then proceeding as in the proof above, we obtain in (5.33)

$$
\frac{d}{d t} \rho(t) \leq C \rho(t)
$$

with some constant $C$. Consequently $\rho(t) \leq 0$. We have shown the following result.

Proposition 5.4 The operator $\Sigma$ has at most one fixed point in $\mathbb{S}\left(t^{\prime}, L, M\right)$.

Finally we can give the proof of Theorem 3.3

Proof of Theorem 3.3 According to Lemmas 4.3 and 5.3, the Schauder fixed point theorem applies to the operator $\Sigma$ on $\mathbb{S}\left(t^{\prime}, L, M\right)$ for appropriate choices of $L, M$ and $t^{\prime}$, i.e. $\Sigma$ has a fixed point $(r, S, T, X)$ in $\mathbb{S}\left(t^{\prime}, L, M\right)$. The regularity conclusions of Theorem 2.2 applied to this fixed point show immediately that

$$
r, S, T \in C^{1}\left(\left[0, t^{\prime}\right] ; H^{1}(0,1)\right) \cap C\left(\left[0, t^{\prime}\right] ; H^{2}(0,1)\right) \cap \mathbb{B} \mathbb{R}\left(0, t^{\prime} ; 0,1\right) .
$$

Moreover, by Proposition 5.4, this is the only fixed point in $\mathbb{S}\left(t^{\prime}, L, M\right)$. When we define the velocity $v$ by

$$
v=w(r, T, X),
$$

then we readily obtain

$$
v \in C^{1}\left(\left[0, t^{\prime}\right] ; H^{2}(0,1)\right) \cap C\left(\left[0, t^{\prime}\right] ; H^{3}(0,1)\right) .
$$

It is clear that $r, S, T, v$ satisfy Eqs. (1.1), (1.3) and (1.4) together with the boundary/initial conditions. The structure of these equations and the regularity properties of $r, S, T, v$ imply then also that

$$
r, S, T \in C^{2}\left(\left[0, t^{\prime}\right] ; L^{2}(0,1)\right) \text { and } X \in C^{2}\left[0, t^{\prime}\right] .
$$


(Actually, we even have $X \in C^{3}\left[0, t^{\prime}\right]$.) Consequently,

$$
v \in C^{2}\left(\left[0, t^{\prime}\right] ; H^{1}(0,1)\right) \text {. }
$$

To see that Eq. (1.2) holds true, we note that $u=r^{2}(S-T)$ is a solution of the boundary-initial value problem

$$
\begin{aligned}
& u_{t}+v u_{z}+\left(2 v_{z}+\frac{1}{\mathrm{We}}\right) u=-3 v_{z} r^{2}\left(T+\frac{1}{\mathrm{We}}\right), \\
& u(t, 0)=-X(t), \quad u(0, z)=-X(0)=\left(r^{0}(z)\right)^{2}\left(S^{0}(z)-T^{0}(z)\right) .
\end{aligned}
$$

However, since $v_{z}$ satisfies

$$
v_{z}=\frac{\mathrm{We} X_{t}+X}{-2 \mathrm{We} X+3 r^{2}(1+\mathrm{We} T)},
$$

$\tilde{u}(t, z)=-X(t)$ is readily seen to be a solution of problem (5.41)-(5.42) as well. Using a standard energy argument, we immediately conclude that solutions of this boundary-initial value problem are unique. Hence Eq. (1.2) holds.

Finally, if $(r, v, S, T)$ is a solution of Eqs. (1.1)-(1.11) with the regularity required in (3.31), then $v$ satisfies Eq. (5.37) with $X(t)=T(t, 0)$. Consequently, $(r, S, T, X)$ is a fixed point of the Schauder map $\Sigma$ on some set $\mathbb{S}\left(t^{\prime}, L, M\right)$. Because of Proposition 5.4, uniqueness is established. This concludes the proof.

\section{Conclusion}

In this work we have given an existence, uniqueness and regularity result for the equations of isothermal fiber spinning for a viscoelastic liquid modeled by the constitutive theory of the upper-convected Maxwell fluid. The proofs were based on energy estimates, a compactness argument and the Schauder fixed point theorem. The main difficulty in the existence proof was due to the fact that only one boundary condition was given for the elastic stresses at the inlet. This issue was addressed by introducing the undetermined boundary stress as an unknown of the problem and as a variable in the solution map. The resultant solution of the governing equations had sufficient smoothness to allow classical derivatives both in time and space.

It is easily seen that, instead of prescribing the radial stress component, we could have imposed an axial stress boundary condition or a condition involving both stress components (such as the ratio of the two) as long as not both stresses are given explicitly at the inlet. For such changes or for nonconstant boundary data the existence and uniqueness results remain correct with minor modifications of the proofs.

Acknowledgment. The authors acknowledge financial support through NSFGrant DMS 0709197. They also express their gratitude to the Institute for Mathematics and its Applications at the University of Minnesota for its hospitality during summer 2008 (DK) and fall 2009 (TH). 


\section{References}

[1] R. B. Bird, R. C. Armstrong, and O. Hassager, Dynamics of Polymeric Liquids, volume 1, second edition. Wiley, New York 1987.

[2] M. G. Forest and Q. Wang, Dynamics of slender viscoelastic free jets, SIAM J. Appl. Math. 54 (1994), 996-1032.

[3] T. Hagen, On viscoelastic fluids in elongation. In Advances in Mathematics Research, vol. 1, G. Oyibo, ed., Nova Science, New York 2002.

[4] T. Hagen, Jeffreys fluids in forced elongation, J. Math. Anal. Appl. 288 (2003), 634-645.

[5] T. Hagen and M. Renardy, On the equations of fiber spinning in nonisothermal viscous flow. In Topics in Nonlinear Analysis. The Herbert Amann Anniversary Volume, J. Escher and G. Simonett, eds., Birkhäuser, Basel 1999.

[6] T. Hagen and M. Renardy, Non-adiabatic elongational flows of viscoelastic melts, Z. Angew. Math. Phys. 51 (2000), 845-866.

[7] M. A. Matovich and J. R. A. Pearson, Spinning a molten threadline steady-state isothermal viscous flows, Ind. Eng. Chem. Fundam. 8 (1969), $512-520$

[8] T. C. Papanastasiou, C. W. Macosko, L. E. Scriven and Z. Chen, Fiber spinning of viscoelastic liquid, AIChE J. 33 (1987), 834-842.

[9] T. C. Papanastasiou, V. D. Dimitriadis, L. E. Scriven, C. W. Macosko and R. L. Sani, On the inlet stress condition and admissibility of solution of fiber-spinning, Adv. Polym. Tech. 15 (1996), 237-244.

[10] M. Renardy, Effect of upstream boundary conditions on stability of fiber spinning in the highly elastic limit, J. Rheol. 46 (2002), 1023-1028.

[11] J. R. A. Pearson, Mechanics of Polymer Processing. Elsevier, London 1985. 